\title{
CORRIGENDUM
}

\section{Prenatal (non)treatment decisions in extreme prematurity: evaluation of decisional conflict and regret among parents}

R Geurtzen, J Draaisma, R Hermens, H Scheepers, M Woiski, A van Heijst and M Hogeveen

Correction to: Journal of Perinatology advance online publication, 15 June 2017; doi:10.1038/jp.2017.90

In the 'Decision-making information' section of Table 1, the $n$ values for 'Joint decision parent(s) and doctor, parental opinion decisive' should be 12/54, not 13/54, and the percentage is therefore 22 instead of 23. The $n$ values for 'Joint decision parent (s) and doctor, both equally decisive' should be 16/54, not $17 / 54$. These changes also affect the last sentence of the first paragraph in the Results section ('According to the parents, most decisions were made together by the doctor and parents (62\%)'); the percentage should be $61 \%$. The corrections to the percentages are slight and do not affect the conclusions of the paper. A corrected version of Table 1 is shown below. The authors regret the errors.

Table 1. Background of the participants

\begin{tabular}{|c|c|c|}
\hline & $\mathrm{n}$ & $\%$ \\
\hline \multicolumn{3}{|l|}{ Demographics of the parents } \\
\hline \multicolumn{3}{|l|}{ Survey filled in by } \\
\hline Both parents & $39 / 61$ & 64 \\
\hline Mother only & $16 / 61$ & 26 \\
\hline Father only & $5 / 61$ & 8 \\
\hline Unknown & $1 / 61$ & 2 \\
\hline \multicolumn{3}{|l|}{ Highest degree of education } \\
\hline Low (maximal secondary education) & $0 / 61$ & 0 \\
\hline Medium (post secondary non academic) & $18 / 61$ & 30 \\
\hline High (academic/college or university) & $35 / 61$ & 57 \\
\hline Unknown & $8 / 61$ & 13 \\
\hline Median age of the mother (at delivery) & $\begin{array}{c}31.5 \\
\text { years }\end{array}$ & $\begin{array}{l}(\mathrm{IQR} 27- \\
36 \text { years) }\end{array}$ \\
\hline \multicolumn{3}{|l|}{ Perinatal data } \\
\hline Any prior pregnancies & $21 / 54$ & 39 \\
\hline Prior miscarriage & $5 / 54$ & 9 \\
\hline $\begin{array}{l}\text { Prior extreme premature birth (GA 24- } \\
26 \text { weeks) }\end{array}$ & $1 / 54$ & 2 \\
\hline Prior premature birth (GA 27-37 weeks) & $2 / 54$ & 4 \\
\hline Prior stillborn child & $3 / 54$ & 6 \\
\hline \multicolumn{3}{|l|}{ Timing of the counseling conversation } \\
\hline At day of birth & $13 / 48$ & 27 \\
\hline 1 day before birth & $2 / 48$ & 4 \\
\hline 2-3 days before birth & $13 / 48$ & 27 \\
\hline$>3$ days before birth & $16 / 48$ & 33 \\
\hline Do not remember & $4 / 48$ & 8 \\
\hline
\end{tabular}

Table 1. (Continued)

\begin{tabular}{|c|c|c|}
\hline & $n$ & $\%$ \\
\hline \multicolumn{3}{|l|}{ Decision-making characteristics } \\
\hline \multicolumn{3}{|l|}{ Decision made on initiating care } \\
\hline Active treatment & $46 / 55$ & 84 \\
\hline Comfort care & $5 / 55$ & 9 \\
\hline Other (for example, wait and see) & $4 / 55$ & 7 \\
\hline \multicolumn{3}{|l|}{ Outcome (in case of active treatment) } \\
\hline Survivor & $24 / 50$ & 48 \\
\hline Death & $22 / 50$ & 44 \\
\hline $\begin{array}{l}\text { Both (twin pregnancy with one survivor } \\
\text { and one deceased) }\end{array}$ & $4 / 50$ & 8 \\
\hline \multicolumn{3}{|l|}{ Self-reported child morbidity (in case of survival) } \\
\hline $\begin{array}{l}\text { Any physical disabilities (minor-major- } \\
\text { severe) }\end{array}$ & $\begin{array}{l}6-0- \\
0 / 26\end{array}$ & $23-0-0$ \\
\hline $\begin{array}{l}\text { Any intellectual disabilities (minor-major- } \\
\text { severe) }\end{array}$ & $\begin{array}{l}2-0- \\
1 / 27\end{array}$ & $7-0-4$ \\
\hline \multicolumn{3}{|l|}{ Decision-making information } \\
\hline \multicolumn{3}{|c|}{ Persons deciding whether or not to initiate active care } \\
\hline Parent(s) & $6 / 54$ & 11 \\
\hline $\begin{array}{l}\text { Joint decision parent(s) and doctor, } \\
\text { parental opinion decisive }\end{array}$ & $12 / 54$ & 22 \\
\hline $\begin{array}{l}\text { Joint decision parent(s) and doctor, both } \\
\text { equally decisive }\end{array}$ & $16 / 54$ & 30 \\
\hline $\begin{array}{l}\text { Joint decision parent(s) and doctor, } \\
\text { doctors' opinion decisive }\end{array}$ & $5 / 54$ & 9 \\
\hline Doctor & $10 / 54$ & 18 \\
\hline Do not remember & $3 / 54$ & 6 \\
\hline $\begin{array}{l}\text { Other (for example, 'obvious' or } \\
\text { 'unclassifiable') }\end{array}$ & $2 / 54$ & 4 \\
\hline $\begin{array}{l}\text { Parents who felt like they had a choice on } \\
\text { whether or not to initiate active treatment for } \\
\text { their baby (\% yes) }\end{array}$ & $43 / 54$ & 80 \\
\hline
\end{tabular}

Abbreviations: GA, gestational age; IQR, interquartile range. 